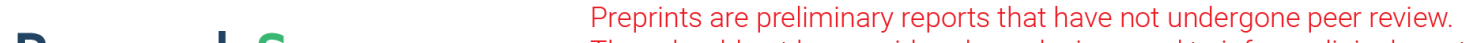 They should not be considered conclusive, used to inform clinical practice, or referenced by the media as validated information. \\ Low NOX1 Expression in The Cytoplasm of GISTs Correlates With Poor Prognosis
}

\section{Wei Wang}

Nantong University Affiliated Hospital

\section{Wenjun You}

the second people's hospital of nantong

\section{Fang Huang}

Nantong University Affiliated Hospital: Affiliated Hospital of Nantong University

\section{Xin He}

Nantong University Affiliated Hospital: Affiliated Hospital of Nantong University

\section{Ao Yuan}

Nantong University Affiliated Hospital: Affiliated Hospital of Nantong University

Huijun Zhu ( $\nabla$ ntzhuhuijun@126.com )

Nantong University Affiliated Hospital

\section{Research}

Keywords: NADPH oxidase I, Neoplasm, Immunohistochemistry, Gastrointestinal Stromal Tumor, GIST

Posted Date: September 18th, 2020

DOl: https://doi.org/10.21203/rs.3.rs-77463/v1

License: (c) (i) This work is licensed under a Creative Commons Attribution 4.0 International License.

Read Full License 


\title{
Low NOX1 expression in the cytoplasm of GISTs correlates with poor prognosis
}

\author{
Wei Wang ${ }^{1 *}$, Wenjun You ${ }^{2 *}$, Fang Huang ${ }^{1}$, Xin He ${ }^{1}$, Ao Yuan ${ }^{1}$, Huijun Zhu ${ }^{1}$ \\ ${ }^{1}$ Department of Pathology, Nantong University Affiliated Hospital, Nantong, Jiangsu 226001, \\ China \\ ${ }^{2}$ Department of Geriatrics, The Second People's Hospital of NanTong, Nantong, Jiangsu \\ 226002, China \\ *Authors' Contributions; Wei Wang and Wenjun You contributed equally to the \\ manuscript. \\ Correspondence should be addressed to Huijun Zhu; ntzhuhuijun@126.com
}

\begin{abstract}
Objective: To investigate the expression effect and biological significance of NADPH oxidase 1 (NOX1) on the growth of human Gastrointestinal stromal tumor (GIST) at a protein level and the correlation between its expression level and patient prognosis.
\end{abstract}

Methods: We enrolled a total of 146 patients confirmed by histopathology to be GIST tumors. Immunohistochemistry(IHC) analysis with GIST tissue microarray was used to assess NOX1 level. The relativity study between NOXl expression level and GIST patient prognosis was evaluated. NOX1 mRNA expression was subsequently demonstrated using online database Oncomine.

Results: Significance association with mitotic index $\left(\chi^{2}=9.476, \mathrm{p}=0.009\right)$ and grade $\left(\chi^{2}=11.392, \mathrm{p}=0.010\right)$ was observed for low NOX1 cytoplasm staining of GIST cells and poor performance status in 78 of $146(53.42 \%)$ GIST tissues, while for GIST patients with high expression of NOX1, there was no significant survival difference in 
disease progression. By univariate analysis, prognosis of GIST patients for overall survival (OS) rates were significantly positively correlated with NOX1 expression level $\left(\chi^{2}=2.429, \mathrm{p}=0.014\right)$, tumor diameter $\left(\chi^{2}=2.048, \mathrm{p}=0.004\right)$, mitotic index $\left(\chi^{2}=2.665, \mathrm{p}<0.001\right)$, and grade $\left(\chi^{2}=3.162, \mathrm{p}=0.001\right)$. Moreover, multivariate analyses demonstrated that low cytoplasm expression of NOX1 $\left(\chi^{2}=2.144, \mathrm{p}=0.046\right)$ and mitotic index $\left(\chi^{2}=2.513, \mathrm{p}=0.001\right)$ were striking related with OS rates, the difference was statistically significant. Kaplan-Meier survival curves further exhibited that low NOX1 cytoplasm expression and advanced mitotic index had a more unfavorable prognosis in GIST patients.

Conclusion: These conclusions indicate that the degree of malignancy of GIST patients is closely related to NOX1 cytoplasm expression and mitotic index, both of which may be taken as an important role in the occurrence and development of GIST. It may act as an unfavorable predictive marker, indicating a new approach of gene therapy for GIST.

Key words: NADPH oxidase 1; Neoplasm; Immunohistochemistry; Gastrointestinal Stromal Tumor, GIST

\section{Introduction}

It is well known that Gastrointestinal stromal tumors (GIST) are the extremely common human sarcomas that mainly arises in the gastrointestinal tract, with 10-15 million new cases diagnosed annually around the world [1]. Malignant GISTs account 
for approximately $30 \%$ of all such GI tumors. GISTs most frequently arise from the stomach followed by the small and then large intestine, and therefore have various clinical presentations such as abdominal pain, bowel obstruction, blood in the stool, or palpable mass [2]. Clinicopathological features including tumour size, mitotic rate and location, were closely associated with GIST patients overall survival (OS) $[3,4,5]$. Function mutations in KIT or platelet-derived growth factor receptor (PDGFR) genes lead to oncogenic gain of GISTs and constitutive activation of the tyrosine kinase receptor. To date, complete surgical resection and tyrosine kinase inhibitors remains the primary treatment measure for localized GIST [6].

In recent years, increasing understanding of GIST biology has enabled better diagnosis and treatment of GIST patients based on their histologic characteristics and molecular genetics. CD117 protein is identified as an commonly present immunohistochemical (IHC) marker for GIST. Moreover, the discovery of the precursors for GIST that GIST might be derived from the interstitial Cajal cells $[7,8]$. Both of IHC and molecular analysis constitute the gold standard of diagnosis [9], which causes GIST to become a chronic disease and prolongs OS of GIST patients after successful treatment.

However, the potential toxic side effects of conventional treatment are substantial. Tumor malignancy is indispensable to the validation of promising therapeutic targets. Monoclonal antibodies against KIT/PDGFR and resistant to many chemotherapeutics will be a problem of treatment in GIST $[10,11,12]$. whereas c-KIT mutations are detected by genetic analysis approximately final duration of adjuvant therapy and 
whatever percent it remains uncertain. Furthermore, gene testing cannot be performed in the pathology departments of a great deal of hospitals due to limitations in test conditions. Consequently, effective diagnostic strategies that predict tumor recurrence and metastasis were gaining an increasing attention from GIST researchers, additional information of the molecular and genetic biomarkers have to be made to predict the recurrence risk and malignancy potential of GIST which probably result in patients benefit and worth further exploration.

NADPH oxidase (NOX) can promote the production of reactive oxygen species (ROS), specially located in the plasma membrane of phagocytes, which is shown to be significant in body defense by killing invading microbes. So far NOX family (NOX1, NOX3, NOX4, NOX5) of the ROS producer in plasma membrane have been characterised. All of them possess the ability of ROS generation like NOX, though their expression is tissue-specific. The NOX family has arisen as a major origin of ROS induction $[13,14,15]$. Furthermore, NOX family- derived ROS functions as a second messenger molecule to be served as a receptor for nuclear redox and participate in the modulation of cell growth, differentiation and apoptosis. It has shown that ROS can act as signaling molecules to regulate several growth responses including angiogenesis $[16,17,18]$. However, it is acknowledged that angiogenesis is necessary for tumor development and metastasis. Anti-angiogenic therapy is key strategy for the management of various tumors.

Of note, high levels of ROS are found in many cancer cells, such as superoxide and hydrogen peroxide[19]. In the present study, NOXl-based oxidase system has been 
considered a significant correlation with certain cancer and play essential roles in cancer progression, which is related to poor prognosis in certain types of human cancers. Nevertheless, based on the relationship between NOXl expression oxidase system and the clinical details to evaluate its clinicopathological significance in human GIST has not been clearly explored. Thus, with the intention to increase patients' benefits, this review is to determine NOXl associated expression and speculate promising alternative therapeutic targets for treatment of GIST tumor.

\section{Materials and methods}

GIST Patient tissues. We selected a total of 146 GIST patients' tissues from two Department of Clinical Pathology, Affiliated Hospital of Nantong University and Nanjing First Hospital Affiliated to Nanjing Medical University, during the eight-year period (2003-2010). Diagnosis was based on histopathological assessment of GIST and then was validated by positive IHC staining by CD117, the $145 \mathrm{kDa}$ transmembrane glycoprotein KIT and a receptor for stem cell factor. Moreover, other clinical data, including age, sex, tumor diameter, mitotic index, gross classification, tumor location, GIST risk classification, and tumor grade, were acquired from the GIST patients' medical records. In addition, based on the Armed Forces Institute of Pathology (AFIP) Miettinen risk classification criterion, we performed the potential risk classification to examine malignancy [20,21]. Symptomatically treated patients and those received by preoperative radiotherapy or chemotherapy were not included 
in the research, and the human research ethics committee of two hospitals authorized the study protocol.

2.2. Tissue Microarray (TMA) Formation and IHC Detection. A total of 146 formalin fixed and paraffin embedded GIST tissues, were obtained from Affiliated Hospital of Nantong University and Nanjing First Hospital Affiliated to Nanjing Medical University obtained between 2003 and 2010. We performed the TMA System (Quick-Ray, UT06; UNITMA, Korea) in the Department of Clinical Pathology, Affiliated Hospital of Nantong University. As described previously, core biopsies (2-mm in size) were obtained from each paraffin-embedded sample and arranged in prepared paraffin blocks [22].

To clarify the expression of NOX1 in GIST TMAs, IHC analysis was carried out to identify the protein expression of NOX1 in GIST. According to standard IHC protocols, paraffin tissue sections with a thickness of 5 micrometres were dewaxed in $100 \%$ xylene, and then rehydrated in a graded ethanol series for 5 minutes each. Next, the GIST TMAs were simultaneously incubated for 60 minutes with anti-NOX1 antibody (1 : 300 dilution, ab78016; Abcam, Cambridge, MA, USA, this rabbit polyclonal antibody is synthetic peptide derived from the $\mathrm{C}$ terminal of Human NOX1), washed, and then conducted incubation with an anti-rabbit horseradish peroxidase(HRP) conjointed antibody at room temperature. Besides, we utilized phosphate-buffered saline instead of the primary antibody as a negative control.

After that, the density of NOX1-positive cells was defined as four grades: 0 for $0-29 \%, 1$ for $30-59 \%, 2$ for $60-79 \%$, and 3 for $80-100 \%$. Staining intensity of 
NOX1-positive cells was also formulated according to different levels: $0,1,2$, or 3 , ranging from negative, weak, moderate and strong intensity, respectively. The product of the density and intensity score was utilized as the final NOX1 staining score. Finally, the cutoff point of the NOX1 expression score was statistically significant basing on OS and was set utilizing the X-tile software program (the Rimm Lab, Yale University, New Haven, CT) like described previously [23]. The final sum of percentage of NOX1-positive cytoplasm reaction and intensity score jointly represented the NOX1 immunostaining score. The NOX1 immunostaining cases were meticulous scored and evaluated by two board-certified senior pathologists separately who were blinded to the case clinical data in our study, according to the behavior of density and intensity of NOX1-positive cells. The mean score from two pathologists was used as the final immunostaining score.

2.3. Statistical Analysis. The relationship between NOX1 expression and clinicopathological parameters was calculated by 2 tests. Factors associated with OS were assessed by logistic regression. Univariate and multivariate analyses were assessed according to Cox proportional hazards regression models. Additionally, survival curves were performed utilizing the Kaplan-Meier method and compared differences between them using the log-rank test. In this research, we performed all statistical analyses by IBM SPSS Statistics 22.0 (SPSS Inc., Chicago, IL) statistical software.

2.4. Confirmation of NOX1 Expression in GIST. To validate NOX1 expression and its association in GIST, we explored expression of NOX1 mRNA in online database 
Oncomine (https://www.oncomine.org). Three NOX1 datasets were used (Cho Gastric dataset: 90 samples, Detwiller Sarcoma dataset: 54 samples and Segal sarcoma2 dataset: 51 samples).

\section{Results}

3.1. Clinicopathologic details. 73 men and 73 women were collected in this paper. 84 GIST patients were aged $\leq 60$ years, 62 patients were aged $>60$ years. 35 GIST patients had tumors $<5 \mathrm{~cm}$ in diameter, 70 patients had tumors $5-10 \mathrm{~cm}$ in size, while 37 patients had tumors $\geq 10 \mathrm{~cm}$ in diameter. 68 patients had $0-5$ mitotic index, 45 patients had 6-10 mitotic index, and 29 patients had $>10$ mitotic index. 16 patients had single nodules, 22 patients had multiple nodules. As for location, GIST tumors in 77 patients were located in the stomach, 54 patients in the intestines, and 15 patients in other organs. What's more, 34 patients were in the very low to low risk group, 51 patients in the moderate to high risk group, as assessed by AFIP Miettinen risk classification. 46 patients were grade 1,53 patients were grade 2, 27 patients were grade 3, and 14 patients were grade 4 , respectly.

3.2. Location and Expression degree of NOX1 status in GIST by IHC staining. We demonstrated that NOX1 positive staining preferentially localizes to the cytoplasm in human GIST. Representative NOX1 staining patterns by IHC analysis are presented in Figure 1. Based on the cutoff point for NOX1 expression, all tissues were categorized and computed using the X-tile software program. Low NOX1 protein expression with cytoplasm staining of tumor cells was observed in 78 of $146(53.42 \%)$ 
GIST samples and the remaining 68 of $146(46.58 \%)$ of the cases showed high NOX1 protein expression with cytoplasm staining. Furthermore, significant association was observed for low NOX1 expression in 78 (53.42\%) patients and was strongly correlated with mitotic index (per $50 \mathrm{HPFs})(\mathrm{p}=0.009)$, grade $(\mathrm{p}=0.010)$ compared with those who had high NOX1 expression (Figure 1).

\subsection{Relationship between cytoplasm Expression of NOX1 and Clinicopathological} details. The close association between NOX1 expression and clinicopathological details of GIST patients is illustrated in Table 1. Among these parameters, cytoplasm staining of NOX1 was striking related to tumor mitotic index $\left(\chi^{2}=9.476, p=0.009\right)$ and grade $\left(\chi^{2}=11.392, p=0.010\right)$. In contrast, there was no obvious association between NOX1 protein expression and other clinicopathological characteristics, including gender, age, gross classification, tumor diameter, AFIP Miettinen risk classification, and tumor location (Supplementary Table 1).

3.4. Survival Analysis. To further confirm the expression profile of NOX1, univariate Cox's regression analysis revealed factors that significantly influenced survival. Statistically significant differences in OS were showed according to low cytoplasm expression of NOX1 $\left(\chi^{2}=2.429, \mathrm{p}=0.014\right)$, tumor diameter $\left(\chi^{2}=2.048, \mathrm{p}=0.004\right)$, mitotic index $\left(\chi^{2}=2.665, \mathrm{p}<0.001\right)$, and GIST grade $\left(\chi^{2}=3.162, \mathrm{p}=0.001\right)$ (Table 2$)$. All the above-mentioned details were correlated with prognosis of GIST patients for OS rates. In addition, the results showed that there was no significant relationship between NOX1 expression and these other factors, such as Gender, Age Gross 
classification, Tumor position, AFIP Miettinen risk classification related to GIST (Supplementary Table 2). Next, multivariate analyses further evaluated that cytoplasm expression of $\operatorname{NOX} 1\left(\chi^{2}=2.144, \mathrm{p}=0.046\right)$ and mitotic index $\left(\chi^{2}=2.513, \mathrm{p}=0.001\right)$ were closely linked with OS rates (Table 2). Meanwhile, Kaplan-Meier survival curves presented that shorter OS was strongly associated with level of NOX1 expression and a risk of death rose whenever NOX1 expression rose $(p=0.011)$. Those who had low NOX1 expression in tumor, had longer OS compared with high NOX1 expression $(\mathrm{p}=0.011)$, and longer OS of the patients was associated with the low Mitotic index $<5$ HPFs compared to those with high Mitotic index $>5$ HPFs $(p<0.001)$. GIST patients with low NOX1 cytoplasm expression and mitotic index $>5$ had a more unfavorable prognosis (Figures 2 and 3), the result of markedly increased was statistically significant $(\mathrm{p}<0.05)$.

\subsection{Association between NOX1 protein expression and prognosis}

To assess the correlation between NOX1 mRNA expression levels in a large number of GIST samples, we presented data obtained from the Oncomine database, a database of DNA and RNA sequencing information culled from the Gene Expression Library. Three databases including seven analyses were determined by comparing the mRNA expression of NOX1 between GIST and normal tissue. Consistent with our data, and that the expression of NOX1 mRNA was exhibited positively correlation with GIST patients $(\mathrm{p}<0.05)$ (Figure 4). 


\section{Discussion}

Accumulating evidence suggest that NOX is the key enzyme that the earliest known system for producing ROS. Of note, a small amount of ROS can enhance immune defense and participate in the regulation of cell differentiation, proliferation, apoptosis, and intercellular signaling pathways. Excessive ROS can cause oxidative stress, accelerating the pathogenesis of inflammation, fibrosis, and tumor formation. Thus, NOX serve significant roles in cancer initiation and progression and diagnosis. Previous studies indicate that NOX1 are associated with patient survival or drug-resistance in human tumors and may be utilized as promising therapeutic targets[24]. It has been widely reported that NOX1 is a member of NOX family. NOX1-mediated ROS production that regulate exocytosis seems to be vital for cancer development in human cancers, associating with unfavorable metastasis and OS in a number of human cancers. Furthermore, NOX1 has been verified as a biomarker of prognosis in partial human cancers and correlated with the degree of cancer progression as well.

More importantly, recent studies have shown that as one of the main sources of ROS, NOX1 plays key roles in cancer transformation and invasion progression $[25,26]$, and it is overexpressed in various types of cancer such as breast cancer, colon cancer, gastric cancer, stomach cancer and prostate cancer $[25,27,28]$. Then, NOX1 has been proposed to function as an oncogene and a therapeutic target $[29,30]$. In addtion, it is suggested that expression of NOX1 might also be relatively high in human colon epithelial cells compared with that in other normal cell types [31]. 
All the above indicate the oncogenic characteristics of NOX1. Hence, we attempted to focuse on the association between NOX1 expression and certain clinicopathological features of GIST. We also showed that NOX1 is mainly localized at cytoplasm. Here, signicance positive staining of low NOX1 was presented in the cytoplasm of GIST cells (78 of 146). We further carry out the univariate and multivariate cox's survival analysis to associate the low NOX1 expression, the clinicopathological parameters and OS of GIST. As shown in Table 2, low NOX1 status, mitotic index were associated with reduced survival in patients with GIST. NOX1 may promote tumor development. Therefore, low expression of NOX familyderived NOX1 and advanced mitotic index may be found as an independent prognostic factor for GIST patients. Kaplan-Meier survival curves showed that longer OS was strongly associated with a low NOX1 expression and a favorable prognosis for GIST patients. Meanwhile, with the increase in the level of mitotic index (per 50 HPFs), the positive expression rate of NOX1 increased significantly. This paper explored immunoexpression of NOX1 protein and its roles and prognostic in tumors, in order to explore the effective targets for diagnosis and predicte poor prognosis of patients with GIST. Furthermore, we also analysed the relationship between NOX1 expression of GIST patients in the Oncomine databases online resources. It was presented that NOX1 protein and mRNA expression levels were both significantly higher in GIST compared with normal GIST tissues(Figure. 4). Intriguingly, these results were consistent with our data of NOX1 protein expression in the previous research. 
Collectively, novelty of the present findings is that low NOX1 expression is confirmed as a useful prognostic marker in the occurrence and progress of GIST and moreover, a useful predictive marker in patients never receiving preoperative radiotherapy or chemotherapy in all stages of the disease. Conversely, GIST patients with high expression of NOX1, there was no significant survival difference in disease progression. Nevertheless, how effects of NOX is involved in cancer cell proliferation, migration and invasion were not referred to in vitro, whether NOX1 is involved in ROS production remains poorly understood and also requires further investigation. Additionally, the development of inhibitors against NOX and ROS-related redox signaling components as well as understanding the underlying mechanism might provide effective therapeutic strategies for treatment of malignant tumor and define the role of each NOX individual and its regulatory pathways [32]. Significant analysis NOX-1 gene and other NOX isoforms and molecular mechanisms in cancer development remains to be performed in future studies.

Taken together, the conclusions of the present research support that low NOX1 expression is relevant to Mitotic index and unfavorable prognosis in GIST patients. Therefore, low NOX1 may have prognostic value as a tumor biomarker for patients with GIST and act as an effective potential factor for assessing the clinical diagnosis and prognosis of GIST. In future investigations, targeted inhibition of NOX1 could be an attractive strategy and a superior route for the treatment and management of GIST occurrence and development. 


\section{Abbreviations}

NOX1:NADPH oxidase 1; $\quad$ GIST:gastrointestinal stromal tumor;

IHC:immunohistochemistry; OS:overall survival;

ROS:reactive oxygen species; AFIP:Armed Forces Institute of Pathology ;

TMA:tissue microarray

\section{Acknowledgements}

Acknowledgement to the technical staff of Pathology of Nantong University Affiliated Hospital, Jiangsu Province, China.

\section{Funding}

This research received no external funding.

\section{Availability of data and materials}

The datasets used and/or analyzed during the current study are available from the corresponding author upon reasonable request.

\section{Authors' Contributions}

Wei Wang,Wenjun You and Huijun Zhu were responsible for the study conception and design. Wei Wang and Wenjun You were responsible for the acquisition of data. Xin He and Ao Yuan performed the immunostaining techniques. Wei Wang, Wenjun You and Fang Huang were responsible for the analysis and interpretation of data. Wei Wang and Huijun Zhu were responsible for the drafting of the manuscript. All authors read and approved the final manuscript.

\section{Ethics approval and consent to participate}

This study was approved by the human research ethics committees of the Department of Clinical Pathology of the Affiliated Hospital of Nantong University and Nanjing First Hospital Affiliated to Nanjing Medical University, Jiangsu Province, China.

\section{Consent for publication}

Not applicable.

\section{Competing interests}

The authors declare that they have no competing interests.

\section{Author details}

${ }^{1}$ Department of Pathology, Nantong University Affiliated Hospital, Nantong, Jiangsu 226001, China

${ }^{2}$ Department of Geriatrics, The Second People's Hospital of NanTong, Nantong, Jiangsu 226002, China

\section{Conflict of Interests}


All authors declared that they have no conflict of interests.

\section{Funding}

This research received no external funding.

\section{References}

[1] Liu X, Qiu H, Zhang P, et al; China Gastrointestinal Stromal Tumor Study Group (CN-GIST). Prognostic factors of primary gastrointestinal stromal tumors: a cohort study based on high-volume centers. Chin J Cancer Res. 2018;30:61-71.

[2] Schaefer IM, Mariño-Enríquez A, Fletcher JA. What is New in Gastrointestinal Stromal Tumor? Adv Anat Pathol. 2017 Sep;24(5):259-267.

[3] Fletcher C D, Berman J J, Corless C, et al. Diagnosis of gastrointestinal stromal tumors: a consensus approach. Hum Pathol 2002;33.

[4] Markku Miettinen, M, Jerzy Lasota M. Gastrointestinal stromal tumors: Pathology and prognosis at different sites. Semin Diagn Pathol 2006;23:70-83.

[5] Joensuu H. Risk stratification of patients diagnosed with gastrointestinal stromal tumor. Hum Pathol 2008;39:1411-1419.

[6] Siamkouris D, Schloesser M, Yousef A. A rare case of gastrointestinal stromal tumor with a liver metastasis infifiltrating the inferior vena cava and extending to the right atrium with an early recurrence after surgical extraction. Case Rep

Cardiol 2019; 2019:2623403.

[7] Miettinen M, Sarlomo-Rikala M, Lasota J. Gastrointestinal stromal tumors: Recent advances in understanding of their biology. Hum Pathol 1999; 30:1213-1220.

[8] Hirota S, Isozaki K, Moriyama Y, et al. Gain-of-function mutations of c-kit in human gastrointestinal stromal tumors. Science 1998; 279: 577-580.

[9] Chiao-En Wu, Chin-Yuan Tzen, et al. Clinical Diagnosis of Gastrointestinal Stromal Tumor (GIST): From the Molecular Genetic Point of View. Cancers 2019;11:679.

[10] Edris B, Willingham S B, Weiskopf K, et al. Anti-KIT monoclonal antibody inhibits imatinib-resistant gastrointestinal stromal tumor growth. Proc Natl Acad Sci USA 2013;110:3501-3506.

[11] London C A, Gardner H L, Rippy S, et al. KTN0158, a Humanized Anti-KIT Monoclonal Antibody, Demonstrates Biologic Activity against both Normal and Malignant Canine Mast Cells. Clin Cancer Res 2017;23 :2565-2574.

[12] Wagner AJ, Kindler H, Gelderblom H, et al. A phase II study of a human anti-PDGFRalpha monoclonal antibody (olaratumab, IMC-3G3) in previously treated patients with metastatic gastrointestinal stromal tumors. Ann Oncol 2017;28:541-546.

[13] K Griendling, D Sorescu, M Ushio-Fukai. NAD $(\mathrm{P}) \mathrm{H}$ oxidase: role in cardiovascular biology and disease. Circ Res 2000;86:494-501.

[14] C Fleury, B Mignotte, J L Vayssiere. Mitochondrial reactive oxygen species in cell death signaling. Biochimie 2002;84:131-141.

[15] J D Lambeth. NOX enzymes and the biology of reactive oxygen. Nat Rev Immunol 2004;4:181-189. 
[16] J Folkman. Angiogenesis in cancer, vascular, rheumatoid and other disease. Nat. Med 1995;1:27-31.

[17] M Ushio-Fukai. Redox signaling in angiogenesis: role of NADPH oxidase. Cardiovasc Res 2006;71:226-235.

[18] M Ushio-Fukai, R W Alexander. Reactive oxygen species as mediators of angiogenesis signaling: role of $\mathrm{NAD}(\mathrm{P}) \mathrm{H}$ oxidase. Mol Cell Biochem 2004;264:85-97.

[19] T P Szatrowski, C F Nathan. Production of large amounts of hydrogen peroxide by human tumour cells. Cancer Res 1991;51:794-798.

[20] M Miettinen, J Lasota. Gastrointestinal stromal tumors: review on morphology, molecular pathology, prognosis, and differential diagnosis. Archives of Pathology and Laboratory Medicine 2006;130:1466-1478.

[21] M Miettinen, J Lasota. Gastrointestinal stromal tumors: pathology and prognosis at different sites. Seminars in Diagnostic Pathology 2006;23:70-83.

[22] J Huang, H Mei, Z Tang, et al. Triple-amiRNA VEGFRs inhibition in pancreatic cancer improves the effiffifficacy of chemotherapy through EMT regulation. Journal of Controlled Release 2017;245:1-14.

[23] J Huang, J Zhang, H Li, et al. VCAM1 expression correlated with tumorigenesis and poor prognosis in high grade serous ovarian cancer. The American Journal of Translational Research 2013;5:336-346.

[24] Trachootham D, Alexandre J, Huang P. Targeting cancer cells by ROS - mediated mechanisms : a radical therapeutic approach. Nat Rev Drug Discov 2009;8:579-591.

[25] Kamata T. Roles of Nox1 and other Nox isoforms in cancer development. Cancer Sci 2009;100:1382-1388.

[26] Brown DI, Griendling KK. Nox proteins in signal transduction. Free Radic Biol Med 2009;47:1239-1253.

[27] Suh YA, Arnold RS, Lassegue B, et al. Cell transformation by the superoxide-generating oxidase Mox1. Nature 1999;401:79-82.

[28] Tominaga K, Kawahara T, Sano T, et a1. Evidence for cancer associated expression of NADPH oxidase 1(Noxl)-based oxidase system in the human stomach. Free Radic Biol Med 2007;43:1627-1638.

[29] Leto TL, Morand S, Hurt D, et al. Targeting and regulation of reactive oxygen species generation by Nox family NADPH oxidases. Antioxid Redox Signal 2009; 11:2607-2619.

[30] Ushio-Fukai M, Nakamura Y. Reactive oxygen species and angiogenesis: NADPH oxidase as target for cancer therapy. Cancer Lett 2008;266:37-52.

[31] Krause KH. Tissue distribution and putative physiological function of NOX family NADPH oxidases. Jpn J Infect Dis 2004;57:S28-S29.

[32] Masuko Ushio-Fukai, Yoshimasa Nakamura. Reactive oxygen species and angiogenesis:NADPH oxidase as target for cancer therapy. cancer letters 2008;266:37-52. 
Figure 1 Immunohistochemical staining was determined on paraffin-embedded $5 \mu \mathrm{m}$ sections. Representative patterns of NOX1 protein expression in GIST are observed. In GIST tissues, green arrow represents negative cytoplasm staining (A1 and A2), red arrow represents positive cytoplasm staining (B1 and B2). A1 and B1: original magnification $\times 100($ bar $=200 \mu \mathrm{m})$ A2 and B2: $\times 400($ bar $=50 \mu \mathrm{m})$.

Figures 2 Significant correlation was found between OS and level of NOX1 expression.

Figures 3 Significant relationship was found between the OS of GIST with different Mitotic index.

Figure 4 We presented data derived from the Oncomine database. The intensity of color shows the respective mRNA levels of NOX1. The red column means the NOX1 mRNA upregulation.

Table 1 Association of NOX1 expression with clinicalpathological parameters of GIST.

Table 2 Univariate and multivariate analysis of prognostic factors for os in GIST patients. 


\section{Figures}
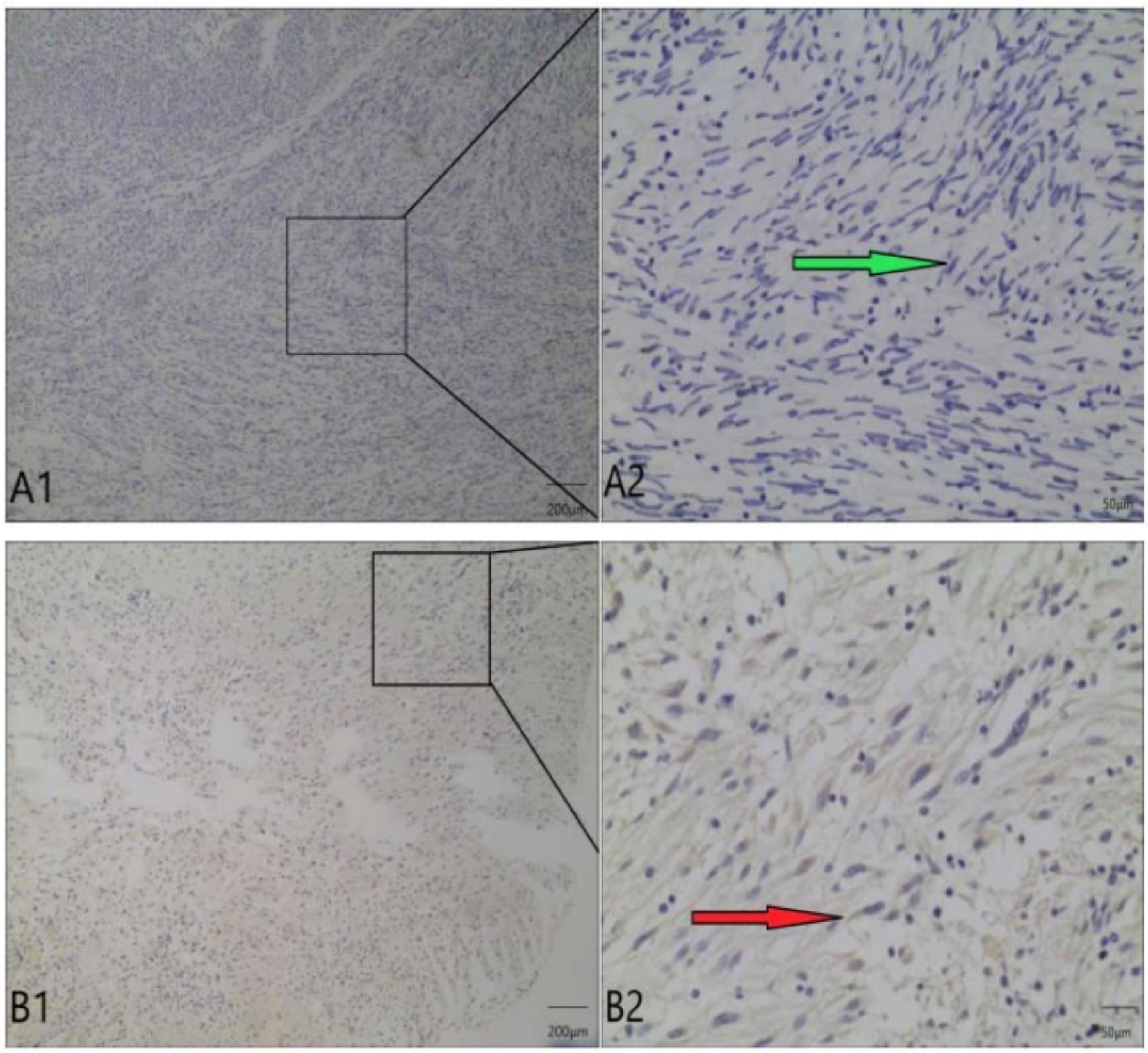

\section{Figure 1}

Immunohistochemical staining was determined on paraffin-embedded $5 \mu \mathrm{m}$ sections. Representative patterns of NOX1 protein expression in GIST are observed. In GIST tissues, green arrow represents negative cytoplasm staining ( $A 1$ and $A 2)$, red arrow represents positive cytoplasm staining (B1 and B2). $A 1$ and B1: original magnification $\times 100($ bar $=200 \mu \mathrm{m}) A 2$ and B2: $\times 400(b a r=50 \mu \mathrm{m})$. 


\section{\&[页面标题]}

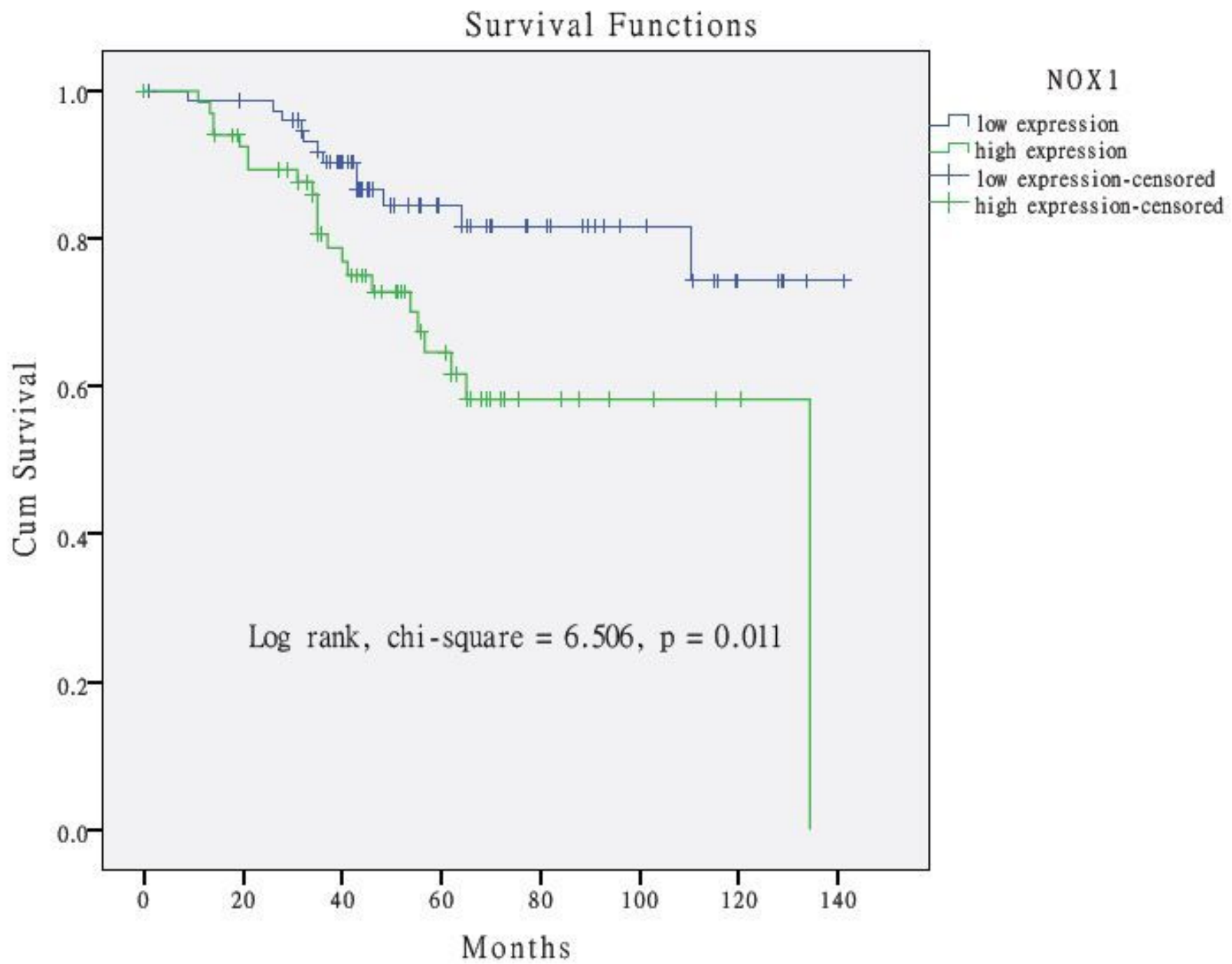

Figure 2

Significant correlation was found between OS and level of NOX1 expression. 
\& 顶面标题]

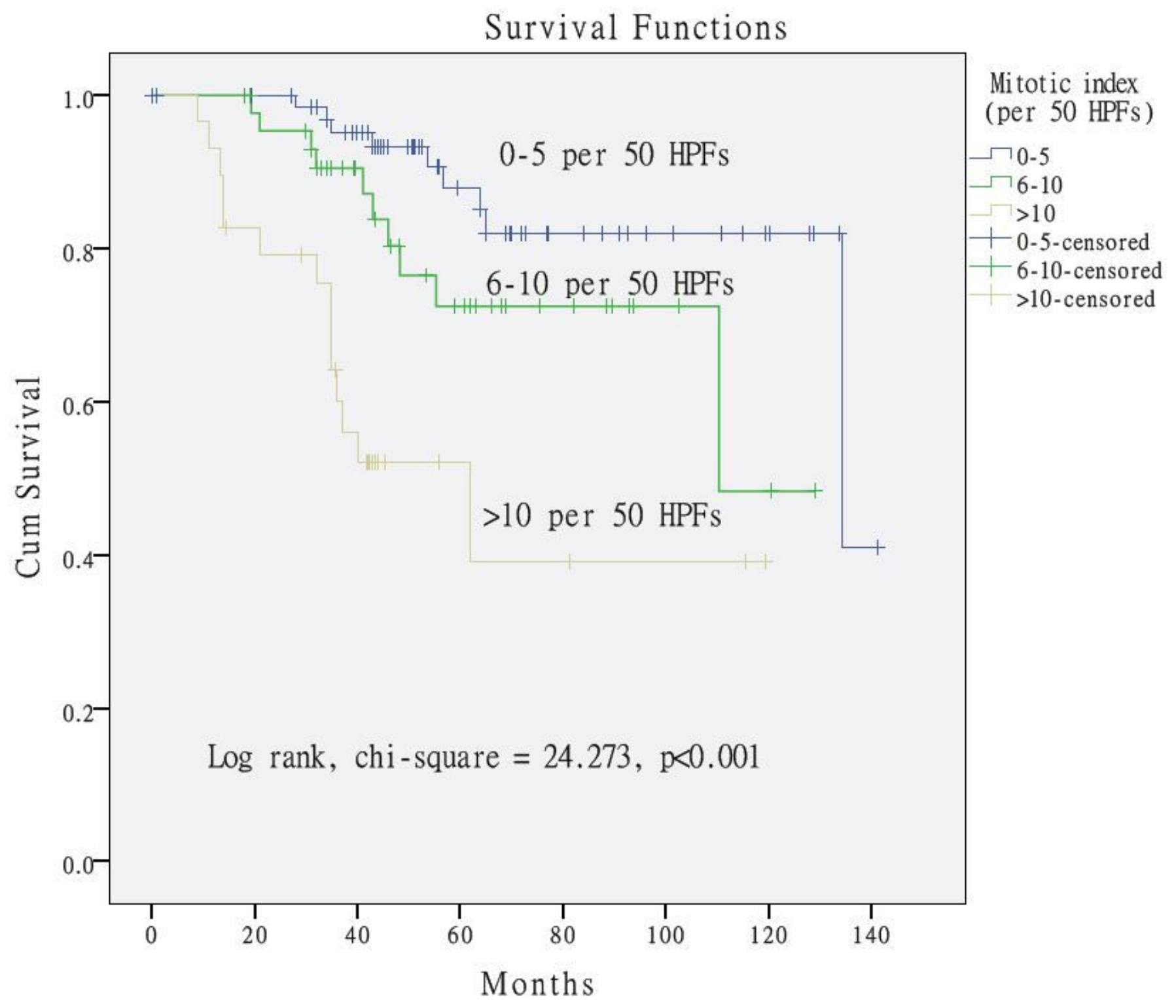

Figure 3

Significant relationship was found between the OS of GIST with different Mitotic index. 


\section{Comparison of NOX1 Across 7 Analyses}

\section{Median Rank COPA Gene \\ $368.0 \quad 5.963$ NOXI}

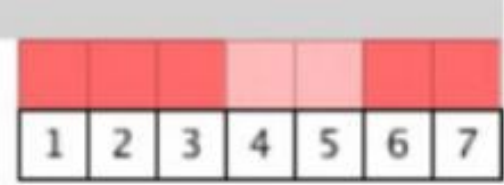

\section{Legend}

1. Outlier 75 th\%

Cho Gastric, Clin Cancer Res, 2011

2. Outlier 90 th\%

Cho Gastric, Clin Cancer Res, 2011

3. Outlier 95 th\%

Cho Gastric, Clin Cancer Res, 2011

4. Outlier 75 th\%

Detwiller Sarcoma, Cancer Res, 2005
5. Outlier 90 th\%

Detwiller Sarcoma, Cancer Res, 2005

6. Outlier 90 th\%

Segal Sarcoma 2, Am J Pathol, 2003

7. Outlier 95 th\%

Segal Sarcoma 2, Am J Pathol, 2003

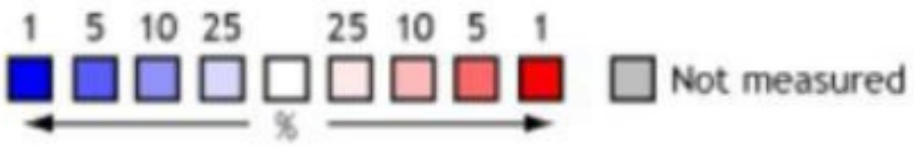

The rank for a gene is the median rank for that gene across each of the analyses.

The COPA score for a gene is its COPA score for the median-ranked analysis.

\section{Figure 4}

We presented data derived from the Oncomine database. The intensity of color shows the respective mRNA levels of NOX1. The red column means the NOX1 mRNA upregulation.

\section{Supplementary Files}

This is a list of supplementary files associated with this preprint. Click to download.

- 9.14Supplementarytable1 and2.docx 